\title{
Lactate kinetics in intensive care unit admissions due to diabetic ketoacidosis
}

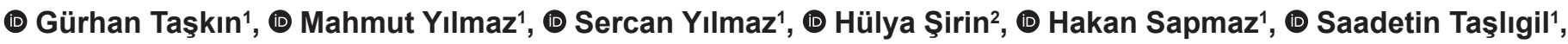 \\ (1) İbrahim Sefa Güneş², (1) Levent Yamanel ${ }^{1}$
}

1 University of Health Sciences Turkey, Gülhane Faculty of Medicine, Department of Intensive Care, Ankara, Turkey

2University of Health Sciences Turkey, Gülhane Faculty of Medicine, Department of Public Health, Ankara, Turkey

\section{Date submitted:}

06.05.2021

Date accepted:

27.07.2021

Online publication date: 15.09.2021

\section{Corresponding Author:}

Gürhan Taşkın, M.D., University of Health Sciences Turkey, Gülhane Faculty of Medicine, Department of Intensive Care, Ankara, Turkey drgurhantaskin@gmail.com

ORCID:

orcid.org/0000-0002-6406-9221

Keywords: Diabetic ketoacidosis, lactate, diabetes mellitus, intensive care unit

\begin{abstract}
Aims: We conducted this study to investigate the clinical significance of lactate kinetics in patients admitted to the intensive care unit (ICU) for diabetic ketoacidosis (DKA).

Methods: This retrospective study was conducted between November 1, 2016, and December 31, 2020. Serial lactate measurements (at the hospital admission, ICU admission, and in 24 hours periods until 48 hours of ICU stay) of the patients admitted to our ICU with a diagnosis of DKA were recorded.

Results: Forty patients were included in the study (mean age: 50.6 19.4 years, female 60\%). Eighty-five percent $(n=34)$ of patients had increased ( $>2 \mathrm{mmol} / \mathrm{L}$ ) blood lactate levels on admission. There was no significant difference between the low (lactate $<4 \mathrm{mmol} / \mathrm{L}$ ) and highlactate (lactate $\geq 4 \mathrm{mmol} / \mathrm{L})$ groups in mortality $(p=0.195)$, ICU stay $(p=0.966)$ and hospital length of stay (LOS) $(p=0.274)$. However, the group with less than $40 \%$ decrease in lactate level from hospital admission to ICU had significantly higher Acute Physiology and Chronic Health Assessment II score [24 (12-46) vs. 18 (2-27), p=0.007], longer ICU stay [5 (1-40) vs. 3 (2-8) days, $p=0.032]$, and higher mortality rate $[6(26.1)$ vs. $0(0.0), p=0.030]$. Additionally, non-survivors $(n=6)$ had significantly higher lactate levels at hospital admission [3.0 (1.3-15.0) vs. 5.1 (3.913.5) $\mathrm{mmol} / \mathrm{L}, \mathrm{p}=0.017$ ], and ICU admission [2.3 (0.6-9.4) vs. $5.0(2.4-16.0) \mathrm{mmol} / \mathrm{L}, \mathrm{p}=0.010$ ] than survivors.
\end{abstract}

Conclusions: Although the ICU LOS and mortality did not differ between initial high and lowlactate groups in the present study, the lactate kinetics, especially in the early treatment period, can guide referral to the ICU level of care and determine the DKA patients at higher risk of death.

\section{Introduction}

Diabetic ketoacidosis (DKA) is primarily characterized by hyperglycemia, ketonemia, and acidosis with an increased anion gap. The number of cases with DKA has been increasing in the last two decades $(1,2)$. The mortality rate of DKA varies across the world due to psychosocial and economic diversities; mainly, it has been reported as less than $1 \%(3,4)$. Although the risk of death is low, intensive care units (ICU) are still the places where these patients are primarily treated, and the prolongation of the ICU stays leads to an increase in hospital costs and ICU overcrowding. In addition, there are no specific criteria for determining whether the patients with DKA should be treated in the ICU or not.
Increased blood lactate concentration, a significant prognostic predictor for many clinical conditions in critically ill patients, is also typical in patients with DKA (5). This may occur due to impaired glucose metabolism with hypoperfusion and poorly understood mechanisms such as the glyoxal pathway and alternative energy substrate in DKA. In addition, there are still controversies regarding the role of increased lactate levels in the course of DKA. Unfortunately, there are insufficient data to suggest that the lactate kinetics in DKA patients may help evaluate treatment response over time and ICU outcomes (6).

This study investigated the clinical significance of lactate kinetics in patients admitted to ICU for DKA. 


\section{Methods}

\section{Study Population}

This retrospective study included patients with DKA hospitalized in the medical ICU between November 01, 2016 and December 31, 2020. The study was approved by the Gülhane Faculty of Medicine, Local Ethics Committee (number: 2020/504, date: 16.06.2016) and performed following the Helsinki Declaration. Patients who had (1) plasma glucose level greater than $250 \mathrm{mg} / \mathrm{dL}$, (2) presence of ketone in serum ( $3 \mathrm{mmol} / \mathrm{L}$ ) or urine $(\geq 2+)$, and (3) plasma bicarbonate level less than or equal to $18 \mathrm{mEq} / \mathrm{L}$ and/or blood $\mathrm{pH}$ less than or equal to 7.30 were included. Patients under 18 years of age and those found to have other causes of lactic acidosis, such as convulsions, use of linezolid or antiretroviral agents, were excluded.

\section{Data Collection}

Demographic characteristics (age, sex), history and type of diabetes mellitus (DM) (type 1 or 2), precipitants for DKA, history of metformin use, comorbidities, and Acute Physiology and Chronic Health Assessment (APACHE) II scores which calculated in the first day of ICU admission were recorded. Systolic blood pressure, diastolic blood pressure, and mean arterial blood pressure at the ICU admission were obtained. Additionally, arterial blood gas analysis results $\mathrm{pH}$, lactate, $\mathrm{HCO}_{3}$ levels), plasma glucose and other laboratory data at hospital admission, hemoglobin A1c (HbA1c) (glycosylated hemoglobin) levels measured during the hospital stay or in the last three months before hospital admission were obtained. Hospital length of stay (LOS), ICU LOS, and in-hospital mortality were recorded.

Patients were grouped into two as low (lactate $<4.0 \mathrm{mmol} / \mathrm{L}$ ) and high-lactate (lactate $\geq 4.0 \mathrm{mmol} / \mathrm{L}$ ) on admission. Additionally, the serial measurements of lactate levels in 24 hours periods until 48 hours of ICU stay, or ICU discharge, whichever came first, were recorded. Laboratory data including plasma glucose, $\mathrm{pH}$, bicarbonate, sodium, and osmolality measured simultaneously with lactate screening (at hospital admission, ICU admission, $24^{\text {th }} \mathrm{hr}, 48^{\text {th }} \mathrm{hr}$ of ICU stay) were obtained.

As there are no published data on the optimal ratio of decrease in lactate levels to predict response to treatment and/ or prognosis in patients with DKA, and the magnitude of lactate reduction following treatment is highly variable in different patient populations (7), we defined a $40 \%$ decrease in lactate levels as the threshold in the early treatment period (between hospital and ICU admissions) for prognosis prediction. Thus, the patients were grouped into two by $40 \%$ or higher decrease in lactate levels from hospital admission to ICU admission. Two previous studies strengthen the feasibility of this approach. Walker et al. (8) suggested a cut-off level of $36 \%$ in lactate clearance in the first 6 hours of treatment for mortality prediction in severe sepsis and septic shock patients who were referred to ICU from the emergency department (ED). In another study, Hernandez et al. (9) reported a $>50 \%$ decrease in lactate levels in the first 6 hours of resuscitation in patients with septic shock. The time (hours) between these two analyses was recorded. The two groups were compared for mortality, APACHE II score, ICU, and hospital LOS.

\section{Statistical Analysis}

Distribution normality for continuous variables was determined by the Shapiro-Wilk test, skewness and kurtosis coefficients, and histogram graphics. The mean (standard deviation) was used to represent parametric continuous variables, and the median (minimum-maximum) was used to represent nonparametric continuous variables. Categorical variables were expressed as numbers (percentage distributions). Independent samples t-test was used to compare parametric variables, and the Mann-Whitney $U$ test was used to compare nonparametric variables. The chi-square test or Fisher's exact test was used for the comparison of categorical variables. Correlations were tested using the Spearman correlation analysis. $\mathrm{P}<0.05$ was considered statistically significant. The data analysis was performed using the IBM Statistical Package for Social Sciences statistics 25.0 (IBM.Corp., Armonk, NY, 2017).

\section{Results}

There were 43 patients with the diagnosis of DKA. After excluding three patients due to missing data, the study population included 40 patients. The mean age of patients was $50.6 \pm 19.4(19-81)$ years, and $24(60 \%)$ patients were female. While $15(37.5 \%)$ patients had type 1 DM, $25(62.5 \%)$ patients had type $2 \mathrm{DM}$. The poor compliance with the treatment $(n=25$, $62.5 \%$ ) was identified as the most common precipitating factor, followed by infections $(n=10,25 \%)$, newly diagnosed $D M(n=1$, $2.5 \%)$ and other causes $(n=4,10 \%)$. Two patients had chronic kidney disease that did not require renal replacement therapy, and three patients had advanced stage malignancy without hepatic involvement. Eighteen patients had been prescribed oral metformin before admission. The median ICU and hospital LOS were 4.0 (1.0-40.0) and 9.0 (2.0-77.0) days, respectively. The overall mortality rate was $15 \%(n=6)$.

Eighty-five percent $(n=34)$ of patients have increased lactate levels $(>2 \mathrm{mmol} / \mathrm{L})$ on admission. High lactate levels (lactate $\geq 4 \mathrm{mmol} / \mathrm{L}$ ) were observed in $16(40 \%)$ of patients. The comparison of demographic, clinical, and laboratory data of lowlactate and high-lactate groups are presented in Table 1. There was no significant difference between the two groups in terms of age, gender, APACHE II score, type of DM, metformin use, blood pressures at ICU admission, duration of DM, glucose, $\mathrm{pH}$, bicarbonate, sodium, urea, creatinine, leukocyte count, hemoglobin, platelet, C-reactive protein, procalcitonin, aspartate 


\begin{tabular}{|c|c|c|c|}
\hline Variables & $\begin{array}{l}\text { Low-lactate } \\
<4.0 \mathrm{mmol} / \mathrm{L} \\
\mathrm{n}=24(60.0 \%)\end{array}$ & $\begin{array}{l}\text { High-lactate } \\
\geq 4 \mathrm{mmol} / \mathrm{L} \\
\mathrm{n}=16(40.0 \%)\end{array}$ & $p$ value \\
\hline Age, years, median (min.-max.) & $51.5(19-81)$ & $53(19-80)$ & $0.413^{\mathrm{r}}$ \\
\hline Gender, female, n (\%) & $16(66.7)$ & $8(50.0)$ & $0.469^{a}$ \\
\hline Type of DM, n (\%) & & & $0.739^{a}$ \\
\hline Type 2 & $14(58.3)$ & $11(68.8)$ & \\
\hline Metformin use, n (\%) & $11(45.8)$ & $7(43.8)$ & $1.000^{\alpha}$ \\
\hline APACHE II, median (min.-max.) & $18.0(2-46)$ & $22.5(12-41)$ & $0.078^{\mathrm{r}}$ \\
\hline \multicolumn{4}{|l|}{ Blood pressures, $\mathrm{mmHg}$} \\
\hline $\mathrm{HbA} 1 \mathrm{c} \pi, \%$, mean $\pm \mathrm{SD}$ & $12.5 \pm 2.4$ & $10.6 \pm 1.5$ & $0.029^{\beta *}$ \\
\hline Duration of $\mathrm{DM}^{\S}$, years, median (min.-max.) & $10.0(0.0-39.0)$ & $8.0(2.0-45.0)$ & $0.510^{r}$ \\
\hline $\mathrm{pH}$, mean $\pm \mathrm{SD}$ & $7.10 \pm 0.1$ & $7.12 \pm 0.1$ & $0.658^{\beta}$ \\
\hline Glucose level, mg/dL, median (min.-max.) & $563.0(159.0-1378.0)$ & $397.0(161.0-837.0)$ & $0.050^{r}$ \\
\hline Bicarbonate, mEq/L, median (min.-max.) & $5.7(2.1-21.9)$ & $9.7(2.4-32.3)$ & $0.136^{\mathrm{r}}$ \\
\hline Sodium, mmol/L, mean \pm SD & $130.0 \pm 7.8$ & $133.9 \pm 4.6$ & $0.091^{\beta}$ \\
\hline Osmolality, mOsm/kg, median (min.-max.) & $300.7(263.1-352.7)$ & $304.6(287.7-349.6)$ & $0.890^{r}$ \\
\hline Hemoglobin, g/dL, mean $\pm S D$ & $13.3 \pm 2.2$ & $12.8 \pm 2.7$ & $0.582^{\beta}$ \\
\hline WBC, $x 10^{3} / \mathrm{mm}^{3}$, mean $\pm S D$ & $16.0 \pm 6.7$ & $16.9 \pm 6.3$ & $0.657^{\beta}$ \\
\hline Bilirubin (total), mg/dL, median (min.-max.) & $0.51(0.10-4.30)$ & $0.49(0.10-3.90)$ & $0.841^{\mathrm{r}}$ \\
\hline CRP, mg/L, median (min.-max.) & $56.63(0.44-421.80)$ & $30.78(2.50-190.00)$ & $0.773^{\mathrm{r}}$ \\
\hline Procalcitonin, ng/mL, median (min.-max.) & $0.70(0.01-16.35)$ & $1.34(0.02-249.00)$ & $0.256^{\vee}$ \\
\hline ICU LOS, days, median (min.-max.) & $4.0(1.0-22.0)$ & $3.5(2.0-40.0)$ & $0.966^{\vee}$ \\
\hline Hospital LOS, days, median (min.-max.) & $9.0(2.0-43.0)$ & $7.5(2.0-77.0)$ & $0.274^{\mathrm{r}}$ \\
\hline In hospital mortality, n (\%) & $2(8.3)$ & $4(25.0)$ & $0.195^{\ddagger}$ \\
\hline
\end{tabular}

aminotransferase, total bilirubin levels, serum osmolality, ICU and hospital LOS or mortality. However, the patients in highlactate group had significantly lower $\mathrm{HbA} 1 \mathrm{c}(12.5 \pm 2.4$ vs. $10.6 \pm 1.5 \%, p=0.029)$ and higher alanine aminotransferase levels [14.0 (4.0-43.0) vs. $21.5(0.0-309.0) \mathrm{U} / \mathrm{L}, \mathrm{p}=0.047$ ] than patients in low-lactate group.

The serial change of lactate levels of the survivors $(n=34)$ and non-survivors $(n=6)$ over time is presented in Figure 1. The median lactate levels were $[3.0(1.3-15.0)$ vs. 5.1 (3.9-13.5) $\mathrm{mmol} / \mathrm{L}$, $p=0.017]$ in survivors and in non-survivors on admission, [2.3 (0.6$9.4)$ vs. $5.0(2.4-16.0) \mathrm{mmol} / \mathrm{L}, \mathrm{p}=0.010]$ at ICU admission, [1.3 (0.3-3.5) vs. $1.4(0.9-11.8) \mathrm{mmol} / \mathrm{L}, \mathrm{p}=0.425]$ at the $24^{\text {th }}$ hour of the ICU stay, and [1.3 (0.5-4.7) vs. $1.6(0.7-5.4) \mathrm{mmol} / \mathrm{L}, \mathrm{p}=0.370]$ at the $48^{\text {th }}$ hour of the ICU stay, respectively.

In patients with or without $\% 40$ or more decrease in lactate levels, there was no significant difference in time from hospital admission to ICU admissions (2.25 vs 3.22 hours, $p=0.556$ ). However, the group with less than $40 \%$ decrease in lactate levels 


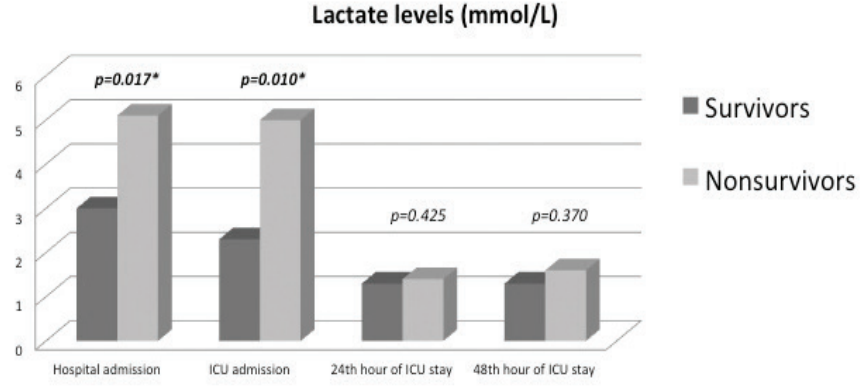

Figure 1. The serial change of lactate levels of survivors and nonsurvivors over time

ICU: Intensive care unit

\begin{tabular}{|c|c|c|c|c|}
\hline & $\begin{array}{l}\text { Total } \\
n=40\end{array}$ & $\begin{array}{l}\text { Decrease } \\
\text { in lactate } \\
<40 \% \\
n=23 \\
(57.5 \%)\end{array}$ & $\begin{array}{l}\text { Decrease } \\
\text { in lactate } \\
\geq 40 \% \\
n=17 \\
(42.5 \%)\end{array}$ & $p$ value \\
\hline $\begin{array}{l}\text { Time between } \\
\text { hospital and } \\
\text { ICU admission, } \\
\text { hours, median } \\
\text { (min.-max.) }\end{array}$ & $\begin{array}{l}2.82 \\
(0.47- \\
76.37)\end{array}$ & $\begin{array}{l}2.25(0.47- \\
39.87)\end{array}$ & $\begin{array}{l}3.22(0.60- \\
76.37)\end{array}$ & $0.556^{\alpha}$ \\
\hline $\begin{array}{l}\text { APACHE II, } \\
\text { median (min.- } \\
\text { max.) }\end{array}$ & $\begin{array}{l}18(2- \\
46)\end{array}$ & $24(12-46)$ & $18(2-27)$ & $0.007^{\alpha, *}$ \\
\hline $\begin{array}{l}\text { ICU LOS, days, } \\
\text { median (min.- } \\
\text { max.) }\end{array}$ & $4(1-40)$ & $5(1-40)$ & $3(2-8)$ & $0.032^{\alpha}, *$ \\
\hline $\begin{array}{l}\text { Hospital LOS, } \\
\text { days, median } \\
\text { (min.-max.) }\end{array}$ & $9(2-77)$ & $11(2-43)$ & $8(2-77)$ & $0.329^{a}$ \\
\hline $\begin{array}{l}\text { In hospital } \\
\text { mortality, n (\%) }\end{array}$ & $6(15)$ & $6(26.1)$ & $0(0.0)$ & $0.030^{\beta}, *$ \\
\hline
\end{tabular}

had significantly higher APACHE II score [24 (12-46) vs 18 (2$27), p=0.007$ ], longer ICU stay [5 (1-40) vs $3(2-8) p=0.032]$, and higher mortality rate [26.1 (6) vs $0(0.0), p=0.030]$ (Table 2).

In correlation analysis, low to moderate significant correlations were found between the initial lactate level and APACHE II score $(r=0.336, p=0.034)$ and $\mathrm{HbA} 1 \mathrm{c}$ level $(r=-0.493$, $p=0.008)$. However, there was no correlation between the initial lactate level and ICU LOS ( $p=0.211)$, hospital LOS $(p=0.603)$, systolic, diastolic and mean arterial blood pressures $(p=0.758$, $p=0.659$ and $p=0.268$, respectively), glucose $(p=0.090), p H$ $(p=0.838)$, bicarbonate $(p=0.456)$, osmolality $(p=0.844)$, and other laboratory tests (not shown).

\section{Discussion}

We hypothesized in the present study that serial changes in lactate level might be more informative than a single admission level in DKA patients. In parallel with previous data (5), there was no difference in mortality and ICU LOS between the on admission high and low-lactate groups in our study. However, it was shown that there was no death among patients who showed more than $40 \%$ decrease in lactate level within the median 3.22 hours before the ICU admission. It was also observed that these patients stayed for a shorter period in the ICU than the patients who could not achieve this decrease. Therefore, it can be thought that decreasing lactate levels in DKA patients, especially in the ED, may help predict the prognosis and decide on the unit (ICU, ED, or general medical ward) where the patient will be managed.

DKA is a common complication of DM, and it constitutes up to $28 \%$ of diabetes-related hospital admissions (10). Many DKA patients are still being treated in ICUs. The main reasons for this preference are the presence of varying degrees of metabolic acidosis, frequent monitoring requirements for blood gas, vital signs, urine output, and serum electrolyte level, and the necessity of intravenous insulin infusion (10-12). However, overcrowding of ICU beds and increased hospital costs must be considered when selecting the ICU level of care to treat these patients. In a study by Marinac and Mesa (13), DKA patients were retrospectively grouped in five severity grades using diastolic blood pressure and some laboratory data (serum bicarbonate, osmolality, anion gap, and base excess). According to their scoring system, the authors reported that more than onethird of all DKA admissions to the ICU were not appropriate in this cohort.

Additionally, previous studies showed that these patients, especially with non-severe DKA, could be safely managed in non-ICU settings such as ED and general medical wards (1416). Nevertheless, the exact criteria for deciding which patient with DKA should be admitted to the ICU have not been fully established yet. The lactate kinetics in the early period of DKA treatment may help this decision.

The inadequate tissue perfusion due to volume depletion was considered the leading cause of elevated lactate levels in DKA patients. Consequently, relative hypoxemia causes an increase in lactate levels by stimulating anaerobic glycolysis $(17,18)$. However, there was no difference in blood pressures between initial high and low-lactate groups in the current study. As explained above, it is thought that tissue hypoxia may not be the only mechanism responsible for elevated lactate levels in DKA patients. Previously, Cox et al. (5) reported a positive correlation between serum lactate and glucose levels in patients with DKA. This finding may indicate a relationship between lactate levels and altered glucose metabolism. On the contrary, 
no correlation was found between initial lactate and glucose levels in the current study, and even a negative correlation was found between $\mathrm{HbA} 1 \mathrm{c}$ and lactate levels.

The reason for the increase in intra-erythrocyte glucose during DKA is that erythrocytes do not need insulin for glucose uptake. The increased intra-erythrocyte glucose is converted first to pyruvate and then to L-lactate through aerobic glycolysis. The remaining amount of glucose is first transformed into methylglyoxal and then to D-lactate with the glyoxalase system. This system also allows the formation of D-lactate in plasma during DKA (19-21). Lu et al. (21) reported that D-lactate levels increased significantly with increased methylglyoxal production during DKA and that high D-lactate levels were significantly correlated with a rising anion gap and decreased bicarbonate levels and thus were associated with DKA severity. The lactate formed during DKA can be used for gluconeogenesis and can be directed to other tissues as an alternative energy substrate due to cellular glucose deficiency. This can be attributed to another mechanism that stimulates the activity of muscle $\mathrm{Na}+\mathrm{K}+$ pumps via the increased catecholamine production due to stress and insulin deficiency in DKA $(22,23)$. As a result, lactate, which increases as an alternative energy substrate for tissues during DKA, can be expected to decrease after the administration of insulin infusion. Therefore, it can be thought that the decrease in lactate level with the initiation of treatment in DKA patients is not only due to rehydration but also due to the decrease in the need for energy substrate with the initiated insulin infusion. The serial measurements of lactate levels may thus be more informative than a single measurement in DKA patients. A systematic review by Vincent et al. (7) on lactate kinetics in critically ill patients suggested that assessing lactate kinetics at 1-2 hour intervals may be more predictive for mortality than baseline values. This recommendation may also be helpful for DKA patients.

The mortality rate in adult DKA patients varies across the world. In the US and UK, the mortality rate has been reported to be less than $1 \%(24,25)$. However, in low-income countries, the in-hospital mortality rate has been reported to be as high as $30 \%$ $(26,27)$. Mortality in adult DKA patients is more likely to result from underlying comorbid diseases, cardiopulmonary complications, or metabolic disorders (hypokalemia/hypoglycemia), which occur during therapy (28). In our study, the overall mortality rate is higher than previously reported. This may be related to the older age of the study population [30\% $(n=12)$ of patients were 65 years of age or older], had a high APACHE II score (20.7 \pm 9.10$)$, and had high $\mathrm{HbA} 1 \mathrm{c}(11.87 \pm 2.32)$ levels indicating long term bad metabolic status.

Additionally, in terms of comorbidities, three patients had advanced stage malignancy without hepatic involvement. Furthermore, a quarter of patients had an infection as a precipitating factor. In a study by Azevedo et al. (12), the inhospital mortality rate was $4 \%$ in cases admitted to the ICU due to DKA, while it was reported as $9 \%$ in severe DKA cases.
Another study by Pasquel et al. (29) revealed that lower bicarbonate levels on admission were significantly related to increased mortality rates. Similarly, in our study, 28 patients had bicarbonate levels less than $10 \mathrm{mEq} / \mathrm{L}$, which could be considered severe DKA. The higher mortality rate observed in our study can be explained by poor comorbid conditions, longterm inadequate medical care, and a high rate of infections.

This study has some limitations. The first is its retrospective design, which may contribute to the results due to other undocumented factors it hides. The lactate metabolism may differ significantly in type $2 \mathrm{DM}$ patients because of more frequent liver involvement than in type $1 \mathrm{DM}$ patients. Evaluation of these two different types of diabetes together may have affected our results. Since our entire study population consisted of critically ill patients and a standard treatment protocol was used independent of diabetes type, we included both types of DM patients in our analyses. In addition, none of the patients included in our study had known chronic liver disease or concomitant fulminant liver failure. The other potential limitation of this study is that the total number of included patients remains small. Additionally, in this study, plasma L-lactate and D-lactate levels were not studied separately. While L-lactate is a marker of tissue hypoxia, $\mathrm{D}$-lactate is a marker of metabolic disarrangements. Therefore, our assessment of the possible pathophysiological mechanisms of changes in lactate kinetics has been limited.

\section{Conclusion}

Although the ICU LOS and overall mortality did not differ between initial high and low-lactate groups, the lactate kinetics, especially in the early treatment period, can guide referral to ICU and determine the DKA patients at higher risk of death. Our results could be confirmed with further studies, including higher numbers of patients to test DKA lactate kinetics.

\section{Ethics}

Ethics Committee Approval: The study was approved by the University of Health Sciences Turkey Gülhane Faculty of Medicine, Local Ethics Committee (number: 2020/504, date: 16.06.2016) and performed following the Helsinki Declaration.

Informed Consent: Retrospective study.

Peer-review: Externally peer-reviewed.

\section{Authorship Contributions}

Design: G.T., M.Y., H.Ş., İ.S.G., Data Collection or Processing: G.T., S.Y., H.S., Analysis or Interpretation: G.T., H.Ş., I.S.G., M.Y., Literature Search: G.T., S.Y., H.Ş., H.S., S.T., Writing: G.T., M.Y., L.Y.

Conflict of Interest: No conflict of interest was declared by the authors.

Financial Disclosure: The authors declared that this study received no financial support. 


\section{References}

1. American Diabetes Association. Economic costs of diabetes in the U.S. in 2012. Diabetes Care. 2013;36:10331046.

2. Centers for Disease Control and Prevention. Diabetes Public Health Resource. Accessed date: April 15, 2021. Available from: http://www.cdc.gov/diabetes/data/

3. Benoit SR, Hora I, Pasquel FJ, Gregg EW, Albright AL, Imperatore G. Trends in Emergency Department Visits and Inpatient Admissions for Hyperglycemic Crises in Adults With Diabetes in the U.S., 2006-2015. Diabetes Care. 2020;43:1057-1064.

4. Gibb FW, Teoh WL, Graham J, Lockman KA. Risk of death following admission to a UK hospital with diabetic ketoacidosis. Diabetologia. 2016;59:2082-2087.

5. Cox K, Cocchi MN, Salciccioli JD, Carney E, Howell M, Donnino MW. Prevalence and significance of lactic acidosis in diabetic ketoacidosis. J Crit Care. 2012;27:132-137.

6. Morgan TJ, Scott PH, Anstey CM, Bowling FG. Hyperlactatemia in diabetic ketoacidosis is common and can be prolonged: lactate time-series from 25 intensive care admissions. J Clin Monit Comput. 2021;35:757-764.

7. Vincent JL, Quintairos E Silva A, Couto L Jr, Taccone FS. The value of blood lactate kinetics in critically ill patients: a systematic review. Crit Care. 2016;20:257.

8. Walker CA, Griffith DM, Gray AJ, Datta D, Hay AW. Early lactate clearance in septic patients with elevated lactate levels admitted from the emergency department to intensive care: time to aim higher? J Crit Care. 2013;28:832-837.

9. Hernandez G, Luengo C, Bruhn A, et al. When to stop septic shock resuscitation: clues from a dynamic perfusion monitoring. Ann Intensive Care. 2014;4:30.

10. Freire AX, Umpierrez GE, Afessa B, Latif KA, Bridges L, Kitabchi AE. Predictors of intensive care unit and hospital length of stay in diabetic ketoacidosis. J Crit Care. 2002;17:207-211.

11. Gershengorn HB, Iwashyna TJ, Cooke CR, Scales DC, Kahn JM, Wunsch $\mathrm{H}$. Variation in use of intensive care for adults with diabetic ketoacidosis*. Crit Care Med. 2012;40:2009-2015.

12. Azevedo LC, Choi H, Simmonds K, Davidow J, Bagshaw SM. Incidence and long-term outcomes of critically ill adult patients with moderate-to-severe diabetic ketoacidosis: retrospective matched cohort study. J Crit Care. 2014;29:971-977.

13. Marinac JS, Mesa L. Using a severity of illness scoring system to assess intensive care unit admissions for diabetic ketoacidosis. Crit Care Med. 2000;28:2238-2241.

14. Karoli R, Fatima J, Salman T, Sandhu S, Shankar R. Managing diabetic ketoacidosis in non-intensive care unit setting: Role of insulin analogs. Indian J Pharmacol. 2011;43:398-401.

15. Cohn BG, Keim SM, Watkins JW, Camargo CA. Does Management of Diabetic Ketoacidosis with Subcutaneous
Rapid-acting Insulin Reduce the Need for Intensive Care Unit Admission? J Emerg Med. 2015;49:530-538.

16. Dunbar LM, Gonzaba WT, DeSoto D, Zaheri K, Sisley $\mathrm{D}$, Thompson $\mathrm{H}$. Aggressive emergency department management of diabetic ketoacidosis can reduce ICU admissions and hospital costs without adversely impacting outcomes. Ann Emer Med. 1999;34:83-84.

17. Fulop M, Hoberman HD, Rascoff JH, Bonheim NA, Dreyer NP, Tannenbaum H. Lactic acidosis in diabetic patients. Arch Intern Med. 1976;136:987-990.

18. Watkins PJ, Smith JS, Fitzgerald MG, Malins JM. Lactic acidosis in diabetes. Br Med J. 1969;1:744-747.

19. Bo J, Li W, Chen Z, Wadden DG, et al. D-lactate: a novel contributor to metabolic acidosis and high anion gap in diabetic ketoacidosis. Clin Chem. 2013;59:1406-1407.

20. Jenei K, Szatmári I, Szabó E, et al. Laktátszintváltozások diabeteses ketoacidosisban és frissen diagnosztizált 1-es típusú diabetes mellitusban [Changes of lactate levels in diabetic ketoacidosis and in newly diagnosed type 1 diabetes mellitus]. Orv Hetil. 2019;160:1784-1790.

21. Lu J, Zello GA, Randell E, Adeli K, Krahn J, Meng QH. Closing the anion gap: contribution of D-lactate to diabetic ketoacidosis. Clin Chim Acta. 2011;412:286-291.

22. James JH, Luchette FA, McCarter FD, Fischer JE. Lactate is an unreliable indicator of tissue hypoxia in injury or sepsis. Lancet. 1999;354:505-508.

23. Bolli G, Cartechini MG, Compagnucci P, Massi-Benedetti $\mathrm{M}$, et al. Attività adrenergica e compenso glicometabolico in pazienti con diabete mellito [Adrenergic activity and glycometabolic compensation in patients with diabetes mellitus]. Minerva Med. 1979;70:3783-3795.

24. Benoit SR, Hora I, Pasquel FJ, Gregg EW, Albright AL, Imperatore G. Trends in Emergency Department Visits and Inpatient Admissions for Hyperglycemic Crises in Adults With Diabetes in the U.S., 2006-2015. Diabetes Care. 2020;43:1057-1064.

25. Gibb FW, Teoh WL, Graham J, Lockman KA. Risk of death following admission to a UK hospital with diabetic ketoacidosis. Diabetologia. 2016;59:2082-2087.

26. Agarwal A, Yadav A, Gutch M, et al. Prognostic Factors in Patients Hospitalized with Diabetic Ketoacidosis. Endocrinol Metab (Seoul). 2016;31:424-432.

27. Ndebele NFM, Naidoo M. The management of diabetic ketoacidosis at a rural regional hospital in KwaZulu-Natal. Afr J Prim Health Care Fam Med. 2018;10:e1-e6.

28. Dhatariya KK, Glaser NS, Codner E, Umpierrez GE. Diabetic ketoacidosis. Nat Rev Dis Primers. 2020;6:40.

29. Pasquel FJ, Tsegka K, Wang H, et al. Clinical Outcomes in Patients With Isolated or Combined Diabetic Ketoacidosis and Hyperosmolar Hyperglycemic State: A Retrospective, Hospital-Based Cohort Study. Diabetes Care. 2020;43:349357. 